TP Periodica Polytechnica

Transportation Engineering

47(4), pp. 268-276, 2019

https://doi.org/10.3311/PPtr. 11377

Creative Commons Attribution (i)

RESEARCH ARTICLE

\section{The Reliability Evaluation of Antiskid Valve of CRH2 EMUs Based on Bayes Method}

\author{
Jianwei Yang $^{1 *}$, Xinghui Qiu ${ }^{1}$, Zhan Chen ${ }^{1}$
}

Received 10 August 2017; accepted 18 September 2017

\begin{abstract}
This paper takes the antiskid valve of braking system as the object of the research. Two parameter Weibull distribution is established through analyzing the operational principle of antiskid valve. According to the Bayesian Reliability, the cumulative failure probability of antiskid valve is calculated. Meanwhile, the posterior distribution is obtained through Markov Chain Monte Carlo. By contrast with these two methods that the reliability of antiskid valve is obtained. According to the least square method, the undetermined parameters of the two parameter Weibull distribution were solved. Then, through the sensitivity analysis, the mathematical model is proved more robust. This paper provides a certain theoretical basis for the assessment of antiskid valve's reliability, and it provides the reference to the other valve in braking system of rail transit vehicle. At the end of the paper, the reliability of the whole antiskid system is calculated through GO method on the basis of the parts' reliability is obtained by Bayes method.
\end{abstract}

\section{Keywords}

Antiskid valve, Weibull distribution, Bayes method, Reliability assessment

\section{Introduction}

China speed up the railway train for the sixth time on April 18,2007 . With the increasing of speed for more than $200 \mathrm{~km} / \mathrm{h}$, $\mathrm{CRH}$ series EMU has become the symbol of China's railway entering the high speed railway. In recent years, Railway passenger turnover accounted for about $40 \%$ of the total turnover of passengers in China. The EMU accident happened on July 23, 2011 makes railroader realize the importance of safety of train. The evaluation of rail vehicle is extremely important for the safety of the operation due to it belongs to the large equipment. Braking system is an important device to ensure the safety of train, and to ensure the comfort of passengers. The study object of this paper is a valve serve in braking system. Through the evaluation of antiskid valve, the weaknesses of this valve can be pointed out. It is laid the foundation for improving the design and boosting its engineering capabilities. Meanwhile, this paper provides a method to other valve that works in EMU trains.

In the field of transit reliability assessment, many research have been done. In reference (Wu et al., 2009), durability test about relay valve of brake system for rail vehicles was done by $\mathrm{Wu}$ Mengling, a fitted failure distribution model is obtained based on experimental data. The failure type and wear-out mechanism of relay valve was analyzed based on estimating the parameter of failure distribution. Zhu Dexin assume that EMU bearing follows two parameter Weibull distribution, through the Bayes method, the failure probability was obtained by estimating the parameter of Weibull distribution. Then the reliability of EMU bearing is obtained through simulation test. In reference (Wang, 2001), the weighted least square method is adopted to fit the three parameter Weibull distribution, then, the reliability and the failure probability of parts is obtained during the high failure rate period. In view of Chinese railway, Wang Lingzhi combine correlation coefficient method with least square method, the reliability of each subsystem is obtained through fitting the three parameter Weibull distribution which based on reference (Wang et al., 2008). Wang Xiaoyan do a research about the reliability evaluation of unit brake which served in the rail transit vehicle through durability test (Li, 2015). Tian Yuanzhen use the virtually expanded sample 
to expand the samples of structure of body bolsters of C70 convertible based on normal distribution.

The reliability of parts of CRH2 EMU attach the importance to the safety of the whole vehicle system. According to the research of statistic. The braking fault of rail transit vehicle most concentrate on the air compressor, electronic brake unit, and all kinds of valve. Traditionally, the evaluation of reliability is the research about life through lots of samples, however, it cost too much funds and time for the tests. Meanwhile, the complex of the system, and the limitation of the number of the test sample of the rail transit vehicle make it even harder. In this paper, the reliability mathematical model is built through accumulating the major failure type of CRH2 EMU antiskid valve. Bayes method is adopted to evaluate the reliability of the valve. More specific, we using Bayes method to calculate the failure probability, simultaneous, Markov Chain Monte Carlo (MCMC) is applied to get the posterior distribution. We combine this two to evaluate the reliability of antiskid valve. Then, in order to validate the model, least square method is adopted to estimating the parameters of Weibull distribution which refers to shape parameter and scale parameter. At last, a calculation example is given to illustrate this method. It provides a certain theoretical basis for the assessment of antiskid valve's reliability. And it provides some references for reliability assessment for other kinds of valve which serve in EMU trains.

\section{Failure type and reliability model of antiskid valve 2.1 Major failure type of antiskid valve}

The rail transit vehicle adopts the electric instruction microcomputer to control the straight braking system, and the regeneration priority control. The microcomputer antiskid device is widely used. The system detecting the slip through the microcomputer, and it issue logic control to antiskid valve. The advantage of this kind of antiskid device is quick in calculation, high monitoring sensitivity, and it can make better use of adhesion (Liu, 2001).

The antiskid device will occur a lot of troubles during the practical application process (Niu, 2010). The failure of the antiskid system will make the sliding out of control, and it may cause very serious consequences. Thus, it is necessary to study on the fault of the antiskid system.

When the train starts to brake, if the braking force is too large to exceed the normal adhesion or the contact condition between the wheel and rail makes the adhesion coefficient decrease, the situation of the adhesive force is less than the braking force is come out, sliding is generate at this time. Sliding can cause a scratch between the wheel tread and the rail surface, at the same time, the braking distance will be increased with the decrease of the braking force. When the antiskid system detecting the sliding of wheels, the brake pressure decreased rapidly by antiskid valve until the adhesive is restored. This kind of antiskid control not only can inhibit the occurrence of sliding effectively, but also can make full use of adhesion in the braking, so that the braking distance of the train can be shortened as far as possible (Yao et al., 2001).

The typical antiskid system mainly consists of three parts: the speed monitoring device, the control unit and the antiskid valve, as shown in the Fig. 1, the antiskid valve is the core component of the antiskid system. Antiskid valve is an important part of antiskid control system of CRH2 EMU. During the operation of EMU, drivers need to make full use of adhesion, however, wheel locked should also be taken into account. To avoid wheel sliding, we control the braking force through controlling the charging and discharging the air in antiskid valve. Its working principle is shown in Fig. 2, during the braking process, if the wheel is not sliding, the valve magnet VM1 and the valve magnet VM2 are both uncharged, and the compressed air enters from $\mathrm{D}$, moves through control cavity. Then come out from $\mathrm{C}$. When the wheel sliding, and braking force should be reduced, the valve magnet VM1 and the valve magnet VM2 are both charged. Meanwhile, VD is closed, so the compressed air can't go through the control cavity, and $\mathrm{VC}$ is open so that the compressed air can release into the atmosphere, thus the braking force is reduced. When the pressure need be holding, the valve magnet VM1 is charged, the compressed air can't inject to the control cavity. Meanwhile, the valve magnet VM2 is uncharged, and VC is closed, so the compressed air can't release into the atmosphere.

The antiskid failure mostly caused by the failure of a component in the antiskid system. As the core component of the antiskid system, the better or not the antiskid valve is determine the antiskid failure happen or not. And the failure of antiskid valve mostly caused by the mechanical failure of the valve body. Based on investigation and research, a few major failure type of antiskid valve is obtained, as shown in Table 1.

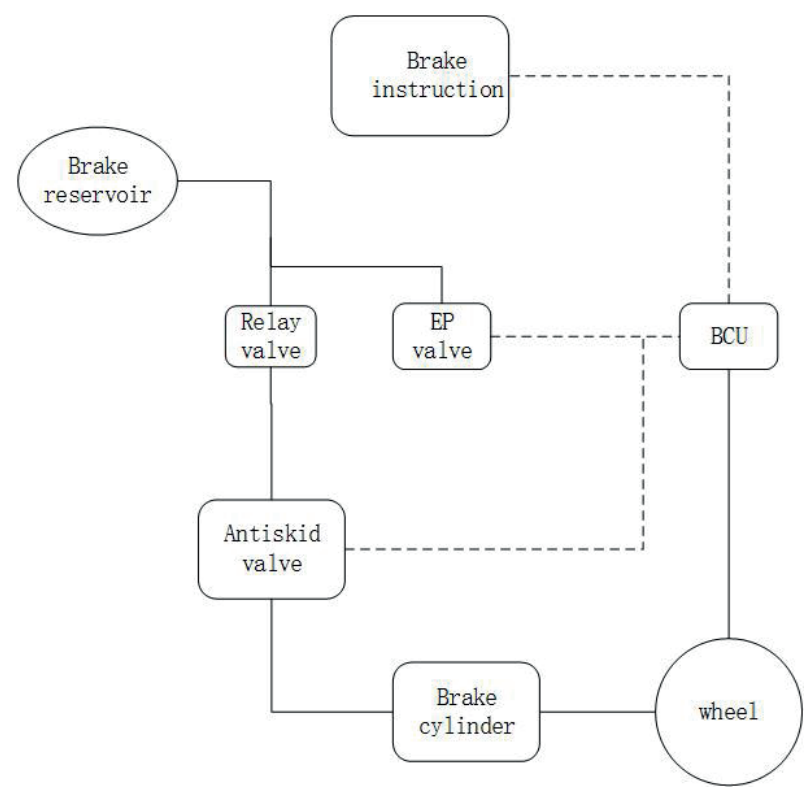

Fig. 1 Schematic diagram of antiskid valve 


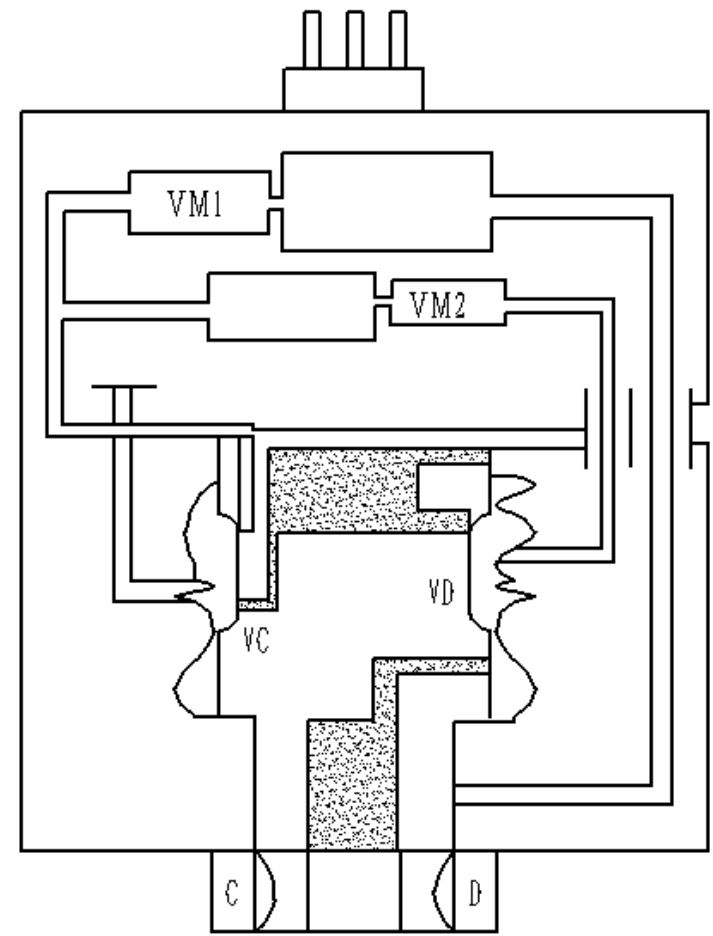

Fig. 2 Working principle of antiskid valve

Table 1 Major failure type of antiskid valve

\begin{tabular}{lll}
\hline Serial number & Parts name & Failure type \\
\hline 1 & Valve body & $\begin{array}{l}\text { Minor scratches on the valve port, and the } \\
\text { tube blockage }\end{array}$ \\
3 & Piston & Damage of rubber sealing ring \\
& Lateral & Minor scratches on the valve port and the \\
& elements & surface of tube \\
valenoid & $\begin{array}{l}\text { The rubber sealing ring of metal sealing } \\
\text { and armature is damaged; crack on the coil }\end{array}$ \\
\hline
\end{tabular}

\subsection{Mathematical model of antiskid valve's reliability}

Through analyzing, it assumes that the antiskid valve's failure type almost follow the wear-out mechanism instead of follow the random failure mechanism. Thus, it assumes that the life of antiskid valve served in CRH2 EMU obey the two parameter Weibull distribution, and the distribution function is given as follows:

$$
F(t)=1-\exp \left[-\left(\frac{t}{\eta}\right)^{\beta}\right]
$$

Where, $F(t)$ is the failure probability of antiskid valve; $t$ is the life of antiskid valve which is a random variable; $\eta$ is the scale parameter, $\beta$ is the shape parameter.

The reliability function of antiskid valve is as follows:

$$
R(t)=1-F(t)=\exp \left[-\left(\frac{t}{\eta}\right)^{\beta}\right]
$$

As shown in (1), in order to obtain the reliability evaluation of antiskid valve's life, the scale parameter $\eta$ and the shape parameter $\beta$ must be certain in the first place. If we follow the traditional way to operate the fatigue tests, then a lot of samples of antiskid valve must be devoted to the experiment, obviously, it is impossible for the dealer of CRH2 EMU antiskid valve to operate this test. Thus, we need to use Bayes method under small samples to solve the problem, that is evaluate the life of antiskid valve served in CRH2 EMU.

\section{The method of Bayesian reliability}

Bayesian reliability analysis is an effective way to evaluate the reliability of many kinds of parts, which includes the topics of modeling, computation, sensitivity analysis, and model checking. During the process of computation, we adopt the information that already know to update the mathematical reliability model that built earlier through Bayes theorem. Bayes school hold that any unknown parameter can take as a random variable, and this variable obey some distribution which is so called prior distribution. The advantage of Bayes method is that we can make full use of prior information to update the mathematical model so that a lot of experiments can be saved.

\subsection{Time censoring zero failure data under small samples}

Due to the limited fund and resources, this paper adopts time censoring test which refers to terminate the test when reach the time preset even there is no failure. During the operation process, we take out $n(n \leq 2)$ antiskid valve as a group to conduct the time censoring test. For this group of antiskid valves, we conduct the experiment for $\mathrm{k}$ times. Assumed that the censoring time is $t_{i}\left(0<t_{1}<t_{2}<\ldots<t_{k}\right)$. And keep the numbers of samples that did not failure as $n_{i}$ during the censoring time $t_{i}$. When the test is finished, the zero failure data is obtained as follows:

$$
\left(t_{i}, n_{i}\right) \quad i=1,2, \ldots, \mathrm{k}
$$

If $s_{i}=n_{i}+n_{i+1}+\ldots+n_{k}$, then the zero failure data of all the samples is:

$$
\left(t_{i}, s_{i}\right) \quad i=1,2, \ldots, \mathrm{k}
$$

When the zero failure data is obtained, the cumulative failure probability need to be calculated. As illustrated before, the cumulative failure probability is taken as a random variable, and it obey some kinds of distribution.

\subsection{Evaluation of cumulative failure probability}

During the $\mathrm{k}$ times time censoring tests of antiskid valve, a cumulative failure probability is obtained which is record as $p_{1}$, $p_{2}, \ldots p_{k}$. Obviously, the cumulative failure probability at the censoring time $t_{i}$ called $p_{i}$. Due to the results of the time censoring test of antiskid valve is failed or non-failed. It accords with the form of binomial distribution. Thus, out of $s_{i}$ samples, there are $r_{i}$ failed, the probability of these failed antiskid valve is: 


$$
P\left(r_{i} \mid p_{i}\right)=C_{s_{i}}^{r_{i}} p_{i}^{r_{i}}\left(1-p_{i}\right)^{s_{i}-r_{i}}
$$

Where, $C_{s_{i}}^{r_{i}}$ refers to there is $r_{i}$ samples failed among the whole $s_{i}$ samples. Particularly, when there is no failure, the probability is simplified to;

$$
P\left(0 \mid p_{i}\right)=\left(1-p_{i}\right)^{s_{i}}
$$

The first step of Bayesian reliability is choosing the right prior distribution for the parameters to be estimated. Under the condition of no historical information and no experts experience, the uniform distribution is chosen as the prior distribution usually for the parameter on some interval. Although the principle of indifference can explain the condition of using the uniform distribution logically, but there is a lot of doubts on this point. In that case, the uniform distribution should be avoided as much as possible. In this paper, we choose the beta distribution Beta $(\alpha, \beta)$ as the prior distribution for the cumulative failure probability $p_{i}$, and the probability density function of beta distribution is as follows:

$$
\begin{gathered}
f(x \mid \alpha, \beta)=\frac{\Gamma(\alpha+\beta)}{\Gamma(\alpha) \Gamma(\beta)} x^{\alpha-1}(1-x)^{\beta-1}, \\
0 \leq x \leq 1, \alpha, \beta>0
\end{gathered}
$$

When the parameter $\alpha, \beta$ in different range of value, the shape of probability density function curve shows different trends. We can see from Fig. 3.

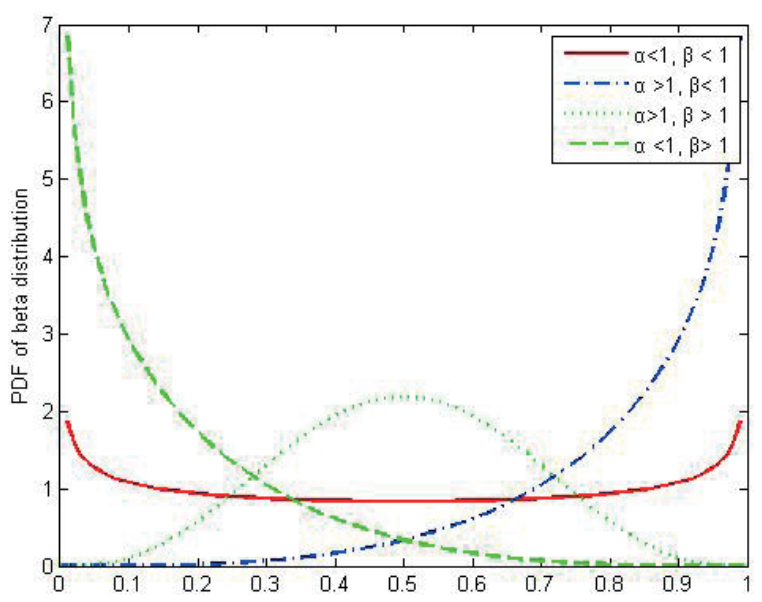

Fig. 3 Probability density function of Beta distribution

By the definition of cumulative failure probability, $p_{1} \leq p_{2} \leq \ldots \leq p_{k}$ can be obtained, according to this, when solving the $p_{2}$, the range of value can be narrowed to $\left(p_{1}, 1\right)$. Thus, the probability density function of beta distribution is no longer the form of (5), it turns out to be incomplete beta distribution, and the form of the incomplete beta distribution is as follows:

$$
f(x)=\frac{\left(x-\theta_{1}\right)^{\alpha-1}\left(\theta_{2}-x\right)^{\beta-1}}{B(\alpha, \beta)\left(\theta_{2}-\theta_{1}\right)^{\alpha+\beta-1}} \quad \theta_{1}<x<\theta_{2}
$$

Where, $B(\alpha, \beta)=\int^{1} x^{\alpha-1}(1-x)^{\beta-1} d x$ is the beta function, and $\alpha>0, \beta>0, \theta_{1}<\theta_{2}^{0}$.

As for the parameter $\alpha$ and $\beta$, it assumed that the parameter $\alpha$ obey the uniform distribution $U(0,1)$. In order to simplify the calculation process, assumed that $\alpha$ equals 0.5 , the parameter $\beta$ obeys the uniform distribution $U(1, c)$, where the hyperparameter $\mathrm{c}$ can be concluded from the experiments of experts.

Through the analysis above, the prior distribution of the cumulative failure probability $p_{i}$ is $\operatorname{Beta}(0,1,0.5, \beta)$. Under the condition of the hyper-parameter $\beta$ obey the uniform distribution $\mathrm{U}(1, \mathrm{c})$, the multilayer prior distribution of the cumulative failure probability is:

$$
f\left(p_{1} \mid c\right)=\int_{1}^{c} \frac{p_{1}^{-0.5}\left(1-p_{1}\right)^{\beta-1}}{B(0.5, \beta)} \frac{1}{c-1} d \beta
$$

According to the Bayesian theorem, the posterior distribution of the cumulative failure probability is as follows:

$$
f\left(p_{1} \mid s_{1}\right)=\frac{f\left(p_{1} \mid c\right) L\left(0 \mid p_{1}\right)}{\int_{0}^{1} f\left(p_{1} \mid c\right) L\left(0 \mid p_{1}\right) d p_{1}}
$$

The Bayesian point estimation is relative to the chosen loss function which the risk value is the smallest, and the most frequently used loss function is $L(\hat{p}, p)=(\hat{p}, p)^{2}$. Where, the $L(\hat{p}, p)$ refers to the risk value of loss when the parameter $\mathrm{p}$ equals $\hat{p}$. under the square loss, the Bayesian evaluation of the parameter $\mathrm{p}$ is the average value of posterior distribution of the cumulative failure probability $p_{i}$. Thus, under the square loss, the Bayesian point estimation of the cumulative failure probability is as follows:

$$
\hat{p}_{1}=E\left(f\left(p_{1} \mid s_{1}\right)\right)=\int_{0}^{1} f\left(p_{1} \mid s_{1}\right) d p_{1}
$$

Where, the $E\left(f\left(p_{1} \mid s_{1}\right)\right)$ refers to the average value of posterior distribution of the cumulative failure probability.

Through analysis above, $p_{1} \leq p_{2} \leq \ldots \leq p_{k}$, thus, it assumed that the prior distribution of the cumulative failure probability $p_{2}$ is $\operatorname{Beta}\left(\hat{p}_{1}, 1,0.5, \beta\right)$, where, the hyper-parameter $\beta$ is obey the uniform distribution $\mathrm{U}(1, \mathrm{c})$, so the multilayer prior distribution of cumulative failure probability $p_{2}$ is as follows:

$$
f\left(p_{2} \mid c, \hat{p}_{1}\right)=\int_{1}^{c} \frac{\left(p_{2}-\hat{p}_{1}\right)^{-0.5}\left(1-p_{2}\right)^{\beta-1}}{B(0.5, \beta)\left(1-\hat{p}_{1}\right)^{\beta-0.5}} \frac{1}{c-1} d \beta
$$

According to the Bayesian reliability, the posterior distribution of the cumulative failure probability $p_{2}$ is as follows: 


$$
f\left(p_{2} \mid s_{2}\right)=\frac{f\left(p_{2} \mid c, \hat{p}_{1}\right) L\left(0 \mid p_{2}\right)}{\int_{\hat{p}_{1}}^{1} f\left(p_{2} \mid c, \hat{p}_{1}\right) L\left(0 \mid p_{2}\right) d p_{2}}
$$

Similarly, the Bayesian point estimation of the cumulative failure probability $p_{2}$ under the square loss is as follow:

$$
\hat{p}_{2}=E\left(f\left(p_{2} \mid s_{2}\right)\right)=\int_{\hat{p}_{1}}^{1} f\left(p_{2} \mid s_{2}\right) d p_{2}
$$

According to the mathematical induction, the prior distribution of the cumulative failure probability $p_{i}$ is $\operatorname{Beta}\left(\hat{p}_{i-1}, 1,0.5, \beta\right)$, and the hyper-parameter $\beta$ is obey the uniform distribution $U$ $(1, \mathrm{c})$. It assumes that the multilayer prior distribution of the cumulative failure distribution $p_{i}$ is as follows:

$$
f=\int_{1}^{c} \frac{\left(p_{i}-\hat{p}_{i-1}\right)^{-0.5}\left(1-p_{i}\right)^{\beta-1}}{B(0.5, \beta)\left(1-\hat{p}_{i-1}\right)^{\beta-0.5}} \frac{1}{c-1} d \beta
$$

Similarly, the posterior distribution of the cumulative failure probability $p_{i}$ is as follows:

$$
f\left(p_{i} \mid s_{i}\right)=\frac{f\left(p_{i} \mid c, \hat{p}_{i-1}\right) L\left(0 \mid p_{i}\right)}{\int_{\hat{p}_{i-1}}^{1} f\left(p_{i} \mid c, \hat{p}_{i-1}\right) L\left(0 \mid p_{i}\right) d p_{i}}
$$

Through the analysis above, it can be concluded the general form of the cumulative failure probability $p_{i}$ based on Bayes method is as follows:

$$
\hat{p}_{i}=E\left(f\left(p_{i} \mid s_{i}\right)\right)=\int_{\hat{p}_{i-1}}^{1} f\left(p_{i} \mid s_{i}\right) d p_{i}
$$

The cumulative failure probability $p_{i}$ which is calculated is the update form of the cumulative failure probability $\hat{p}_{i-1}$, the results of the cumulative failure probability $p_{i}$ is not only relative to the cumulative failure probability $\hat{p}_{i-1}$, but also relative to the number of samples and the parameter $\mathrm{c}$. Thus, this method can make full use of the information of samples and the experience of experts, it makes the results more reasonable.

\section{Markov chain Monte Carlo (MCMC)}

MCMC is a special case of Monte Carlo method, it can introduce the Markov Chain process which is a stochastic process into the simulation of the Monte Carlo. Thus, it can achieve the dynamic simulation of the sampling distribution, eventually. The estimation of the parameter can be obtained by the integration of Monte Carlo. In this paper, the calculation is most about a multilayer Bayes distribution which concludes a hyper-parameter. The process of calculating is relative simple. However, as for the multidimensional integration with many hyper-parameters, the calculating is extremely difficult. The MCMC method is numerical simulation way to solve the complex posterior distribution. The purpose of the application of MCMC method is to verify the reliability of the Bayes method on the one hand, on the other hand, it can lay the foundation of the research which the parameter is improved.

The most common category of MCMC is MetropolisHastings algorithm and the Gibbs sampling. The former one is adopted in this paper, the main idea of Metropolis-Hastings algorithm is: the first step of Metropolis-Hastings algorithm is generating a candidate point, usually, the candidate point differs from the current value of the parameter only in one or two components. The second step is compute the probability that the candidate value will be accepted as the next simulated value in the sequence. And the third step is accepting or reject the candidate point with the probability, if accepted, the candidate turns to be the next simulation data, if not, the value is maintained. The detailed procedure is as follow:

1) Generate the candidate point $x_{(0)}$.

2) Given the proposal distribution $\mathrm{q}\left(x_{(k)}, x_{(k-1)}\right)$, this distribution refers to the probability of the value of $x_{(k)}$ transfer to the value of $x_{(k-1)}$. This probability is also called alternative probability. Based on the current value, extract the $x^{*}$ from the distribution $\mathrm{q}\left(x_{(k)}, x_{(k-1)}\right)$

3) Compute the accept probability $\alpha_{\text {accept }}$.

$$
\alpha_{\text {accept }}=\min \left[1, \frac{p\left(x^{*}\right) q\left(x^{*}, x(k-1)\right)}{p(x(k-1)) q\left(x(k-1), x^{*}\right)}\right]
$$

4) Extract the value of $\alpha^{\prime}$ from $[0,1]$, if $\alpha^{\prime}<\alpha_{\text {accept }}$, then the $x^{*}$ is accepted. If not, $x^{*}$ is rejected, that is $x_{(k)}=x_{(k-1)}$.

5) Repeat the previous step, until the sampling is down.

As for the other kinds of valve in CRH2 EMU braking system, such as relay valve, we can use the same way to evaluate the reliability of it. Relay valve is also the mechanical component of braking system, the failure of it almost caused by the mechanical failure of the valve body. So, it can assume that the mathematical model of the relay valve's reliability is also the two parameter Weibull distribution. And the prior distribution of the cumulative probability is beta distribution which is (6).

As for the parameter $\alpha$ and $\beta$, it assumed that the parameter $\alpha$ obey the uniform distribution $U(0,1)$. In order to simplify the calculation process, assumed that $\alpha$ equals 1 , the parameter $\beta$ obeys the uniform distribution $U(1, d)$, where the hyperparameter $\mathrm{c}$ can be concluded from the experiments of experts.

Through the analysis above, the prior distribution of the cumulative failure probability $p_{i}$ is $\operatorname{Beta}(0,1,1, \beta)$. Under the condition of the hyper-parameter $\beta$ obey the uniform distribution $U(1, d)$, the multilayer prior distribution of the cumulative failure probability is: 


$$
\begin{aligned}
f\left(p_{1} \mid d\right)=\int_{1}^{d} \frac{\left(1-p_{1}\right)^{\beta-1}}{B(1, \beta)} \frac{1}{d-1} d \beta \\
=\frac{1}{d-1}\left[\frac{d\left(1-p_{1}\right)^{d-1}}{\ln \left(1-p_{1}\right)}-\frac{\left(1-p_{1}\right)^{d-1}}{\left(\left(1-p_{1}\right)^{d-1}\right)^{2}}-\frac{1}{\left(1-p_{1}\right)^{d-1}}\right. \\
\left.+\frac{1}{\left(1-p_{1}\right)^{d-1}}\right]
\end{aligned}
$$

According to the Bayesian theorem, the posterior distribution of the cumulative failure probability is as follows:

$$
f\left(p_{1} \mid s_{1}\right)=\frac{f\left(p_{1} \mid d\right) L\left(0 \mid p_{1}\right)}{\int_{0}^{1} f\left(p_{1} \mid d\right) L\left(0 \mid p_{1}\right) d p_{1}}
$$

The Bayesian point estimation is relative to the chosen loss function which the risk value is the smallest, and the most frequently used loss function is $L(\hat{p}, p)=(\hat{p}, p)^{2}$. Where, the $L(\hat{p}, p)$ refers to the risk value of loss when the parameter $\mathrm{p}$ equals $\hat{p}$. under the square loss, the Bayesian evaluation of the parameter $\mathrm{p}$ is the average value of posterior distribution of the cumulative failure probability $p_{i}$. Thus, under the square loss, the Bayesian point estimation of the cumulative failure probability is as follows:

$$
\begin{gathered}
\hat{p}_{1}=E\left(f\left(p_{1} \mid s_{1}\right)\right)=\int_{0}^{1} f\left(p_{1} \mid s_{1}\right) d p_{1}=\int_{0}^{1} \int_{1}^{d} \frac{p_{1}\left(1-p_{1}\right)^{\beta+s_{1}-1}}{(d-1) B(1, \beta)} d \beta d p_{1} \\
/ \int_{0}^{1} \int_{1}^{d} \frac{\left(1-p_{1}\right)^{\beta+s_{1}-1}}{(d-1) B(1, \beta)} d \beta d p_{1}=\left[\left(1+s_{1}\right) \ln \frac{s_{1}+d+1}{s_{1}+2}-s_{1} \frac{s_{1}+d}{s_{1}+1}\right] \\
/\left[d-1-s_{1} \frac{s_{1}+d}{s_{1}+1}\right]
\end{gathered}
$$

Where, the $E\left(f\left(p_{1} \mid s_{1}\right)\right)$ refers to the average value of posterior distribution of the cumulative failure probability.

Through analysis above, $p_{1} \leq p_{2} \leq \ldots \leq p_{k}$, thus, it assumed that the prior distribution of the cumulative failure probability $p_{2}$ is $\operatorname{Beta}\left(\hat{p}_{1}, 1,1, \beta\right)$, where, the hyper-parameter $\beta$ is obey the uniform distribution $U(1, d)$, so the multilayer prior distribution of cumulative failure probability $p_{2}$ is as follows:

$$
\begin{gathered}
f\left(p_{2} \mid d, \hat{p}_{1}\right)=\int_{1}^{d} \frac{\left(1-p_{2}\right)^{\beta-1}}{B(1, \beta)\left(1-\hat{p}_{1}\right)^{\beta}} \frac{1}{d-1} d \beta \\
=\frac{1}{d-1}\left[\frac{d q_{2}^{d-1}}{\ln q_{2}}-\frac{q_{2}^{d-1}}{\ln q_{2}^{2}}-\frac{1}{\ln q_{2}}-\frac{1}{\ln q_{2}^{2}}\right] \\
\text { Where, } q_{2}=\frac{1-p_{2}}{1-\hat{p}_{1}}
\end{gathered}
$$

According to the Bayesian reliability, the posterior distribution of the cumulative failure probability $p_{2}$ is as follows:

$$
f\left(p_{2} \mid s_{2}\right)=\frac{f\left(p_{2} \mid d, \hat{p}_{1}\right) L\left(0 \mid p_{2}\right)}{\int_{\hat{p}_{1}}^{1} f\left(p_{2} \mid d, \hat{p}_{1}\right) L\left(0 \mid p_{2}\right) d p_{2}}
$$

Similarly, the Bayesian point estimation of the cumulative failure probability $p_{2}$ under the square loss is as follow:

$$
\begin{aligned}
& \hat{p}_{2}=E\left(f\left(p_{2} \mid s_{2}\right)\right)=\int_{\hat{p}_{1}}^{1} f\left(p_{2} \mid s_{2}\right) d p_{2} \\
& =\int_{\hat{p}_{1}}^{1} \int_{1}^{d} \frac{p_{2}\left(1-p_{2}\right)^{\beta+s_{2}-1}}{(d-1) B(1, \beta)\left(1-\hat{p}_{1}\right)^{\beta}} d \beta d p_{2} \\
& / \int_{\hat{p}_{1}}^{1} \int_{1}^{d} \frac{\left(1-p_{2}\right)^{\beta+s_{1}-1}}{(d-1) B(1, \beta)\left(1-\hat{p}_{1}\right)^{\beta}} d \beta d p_{1} \\
& =\hat{p}_{1}+\left(1-\hat{p}_{1}\right)\left[\left(1+s_{2}\right) \ln \frac{s_{2}+d+1}{s_{2}+2}-s_{2} \frac{s_{2}+d}{s_{2}+1}\right] \\
& /\left[d-1-s_{2} \frac{s_{2}+d}{s_{2}+1}\right]
\end{aligned}
$$

According to the mathematical induction, the prior distribution of the cumulative failure probability $p_{i}$ is $\operatorname{Beta}\left(\hat{\mathrm{p}}_{\mathrm{i}-1}, 1,1, \beta\right)$, and the hyper-parameter $\beta$ is obey the uniform distribution $\mathrm{U}(1, \mathrm{~d})$. It assumes that the multilayer prior distribution of the cumulative failure distribution $p_{i}$ is as follows:

$$
\begin{gathered}
f\left(p_{i} \mid d, \hat{p}_{i-1}\right)=\int_{1}^{d} \frac{\left(1-p_{i}\right)^{\beta-1}}{B(1, \beta)\left(1-\hat{p}_{i-1}\right)^{\beta}} \frac{1}{d-1} d \beta \\
=\frac{1}{d-1}\left[\frac{d q_{i}^{d-1}}{\ln q_{i}}-\frac{q_{i}^{d-1}}{\ln q_{i}^{2}}-\frac{1}{\ln q_{i}}-\frac{1}{\ln q_{i}^{2}}\right] \\
\text { Where, } q_{i}=\frac{1-p_{i}}{1-\hat{p}_{i-1}}
\end{gathered}
$$

Similarly, the posterior distribution of the cumulative failure probability $p_{i}$ is as follows:

$$
f\left(p_{i} \mid s_{i}\right)=\frac{f\left(p_{i} \mid c, \hat{p}_{i-1}\right) L\left(0 \mid p_{i}\right)}{\int_{\hat{p}_{i-1}}^{1} f\left(p_{i} \mid c, \hat{p}_{i-1}\right) L\left(0 \mid p_{i}\right) d p_{i}}
$$

Through the analysis above, it can be concluded the general form of the cumulative failure probability $p_{i}$ based on Bayes method is as follows:

$$
\begin{aligned}
& \hat{p}_{i}=E\left(f\left(p_{i} \mid s_{i}\right)\right)=\int_{\hat{p}_{i-1}}^{1} f\left(p_{i} \mid s_{i}\right) d p_{i} \\
& =\int_{\hat{p}_{i-1}}^{1} \int^{d} \frac{p_{i}\left(1-p_{i}\right)^{\beta+s_{i}-1}}{(d-1) B(1, \beta)\left(1-\hat{p}_{i-1}\right)^{\beta}} d \beta d p_{i} / \\
& \int_{\hat{p}_{i-1}}^{1} \int_{1}^{d} \frac{\left(1-p_{i}\right)^{\beta+s_{i}-1}}{(d-1) B(1, \beta)\left(1-\hat{p}_{i-1}\right)^{\beta}} d \beta d p_{i} \\
& =\hat{p}_{i-1}+\left(1-\hat{p}_{i-1}\right)\left[\left(1+s_{i}\right) \ln \frac{s_{i}+d+1}{s_{i}+2}-s_{i} \frac{s_{i}+d}{s_{i}+1}\right] \\
& /\left[d-1-s_{i} \frac{s_{i}+d}{s_{i}+1}\right]
\end{aligned}
$$


The cumulative failure probability $p_{i}$ which is calculated is the update form of the cumulative failure probability $\hat{p}_{i-1}$, the results of the cumulative failure probability $p_{i}$ is not only relative to the cumulative failure probability $\hat{p}_{i-1}$, but also relative to the number of samples and the parameter $\mathrm{c}$. Thus, this method can make full use of the information of samples and the experience of experts, it makes the results more reasonable.

\section{The evaluation of the antiskid valve's reliability}

Through the two methods analyzed above, the cumulative failure probability $p_{i}$ is obtained. According to (1), the shape parameter $\beta$ and the scale parameter $\eta$ can be calculated through the weighted least square method which the cumulative failure probability $\mathrm{F}(\mathrm{t})$ is well-known.

Based on the $p_{i}=\mathrm{F}(\mathrm{t})=1-\exp \left[-(t / \eta)^{\beta}\right]$, use the calculated $\hat{p}_{i}$ replace each cumulative failure probability $p_{i}$. And applied the weighted least square method to fitting the parameter, choose the weight coefficient as follows:

$$
\omega_{i}=n_{i} t_{i} / \sum_{i=1}^{k} n_{i} t_{i}(i=1,2, \ldots, k)
$$

It assumed that:

$$
Q(\beta, \eta)=\sum_{i=1}^{k} \omega_{i}\left(p_{i}-1+\exp \left(-\frac{t_{i}}{\eta}\right)^{\beta}\right)^{2}
$$

Take the derivative of (16), then:

$$
\left\{\begin{array}{l}
\frac{\partial Q}{\partial \beta}=\frac{\partial \sum_{i=1}^{k} \omega_{i}\left(p_{i}-1+\exp \left(-\frac{t_{i}}{\eta}\right)^{\beta}\right)^{2}}{\partial \beta}=0 \\
\frac{\partial Q}{\partial \eta}=\frac{\partial \sum_{i=1}^{k} \omega_{i}\left(p_{i}-1+\exp \left(-\frac{t_{i}}{\eta}\right)^{\beta}\right)^{2}}{\partial \eta}=0
\end{array}\right.
$$

Through the calculating, the shape parameter $\beta$ and the scale parameter $\eta$ which makes the (16) the minimum value is obtained as $\hat{\beta}$ and $\hat{\eta}$, that is as follows:

$$
\begin{gathered}
\hat{\beta}=\frac{B-A^{2}}{D-A^{*} C} \\
\hat{\eta}=\exp \left[\frac{B^{*} C-A^{*} D}{B-A^{2}}\right]
\end{gathered}
$$

Where, $\quad A=\sum_{i=1}^{k} \omega_{i} \ln \left(\ln \frac{1}{1-\hat{p}_{i}}\right)$,

$B=\sum_{i=1}^{k} \omega_{i}\left[\ln \left(\ln \frac{1}{1-\hat{p}_{i}}\right)\right]^{2}, \quad C=\sum_{i=1}^{k} \omega_{i} \ln t_{i}$,

$D=\sum_{i=1}^{k} \omega_{i} \ln t_{i}^{*} \ln \left(\ln \frac{1}{1-\hat{p}_{i}}\right)$.
According to (2), when the estimation of the shape parameter and the scale parameter are obtained through weighted least square method, the reliability of antiskid valve at any time can be calculated by (19), which is as follows:

$$
R(t)=\exp \left[-\left(\frac{t}{\hat{\eta}}\right)^{\hat{\beta}}\right]
$$

\section{Numerical examples}

The test is about the antiskid valve severed in CRH2 EMU, 2 samples were taken randomly as a group, and we conduct the time censoring experiment for ten times. According to the maintenance modes of $\mathrm{CRH} 2$ type antiskid valve, that is repaired by suppliers according to the repair scheme, and repair when the mileage is about 1.2 million kilometers, it assumed that the censor time of the test about antiskid valve is 3450 hours.

Since the maintenance cycle of antiskid valve is large, and the failure rate of antiskid valve is low, it's not quick-wear parts. When the censor time is up to $3450 \mathrm{~h}$, there is no failure data, the detail is as Table 2 .

Table 2 Data of time censoring test

\begin{tabular}{lccc} 
Serial number & Censor time $t_{i}$ & Number of sample $n_{i}$ & Non-failure $s_{i}$ \\
\hline 1 & 345 & 2 & 20 \\
2 & 690 & 2 & 18 \\
3 & 1035 & 2 & 16 \\
4 & 1380 & 2 & 14 \\
5 & 1725 & 2 & 12 \\
6 & 2070 & 2 & 10 \\
7 & 2415 & 2 & 8 \\
8 & 2760 & 2 & 6 \\
9 & 3105 & 2 & 4 \\
10 & 3450 & 2 & 2 \\
\hline
\end{tabular}

On the basis of the expert experiences, it assumed that the valve of hyper-parameter $\mathrm{c}$ is 1175 . According to the censor time, number of sample and the non-failure data provided in Table 2. And the Bayesian reliability method, MCMC method analyzed before, the results of the cumulative failure probability is obtained as Table 3 .

Table 3 The results of the cumulative failure probability

\begin{tabular}{lccccc}
\hline Algorithm & $p_{1}$ & $p_{2}$ & $p_{3}$ & $p_{4}$ & $p_{5}$ \\
\hline Bayes & 0.22 & 0.56 & 0.97 & 1.21 & 1.39 \\
MCMC & 0.17 & 0.23 & 1.09 & 1.13 & 1.61 \\
\hline Algorithm & $p_{6}$ & $p_{7}$ & $p_{8}$ & $p_{9}$ & $p_{10}$ \\
\hline Bayes & 1.47 & 1.85 & 2.37 & 2.66 & 3.49 \\
MCMC & 1.69 & 2.34 & 2.23 & 2.85 & 2.55 \\
\hline
\end{tabular}


Through comparing the two method that Table 3 is present, obviously, the results of these two methods is closely. Thus, the accuracy of Bayesian reliability is confirmed which is analyzed before. Simultaneously, it offers the reference to the multidimensional integration with various hyper-parameters to use the MCMC method. To save the space of this paper, the results that conclude from Bayes method is adopted to carry on the illustration.

According to the weighted least square method which is analyzed on chapter 5 , the Bayesian reliability is applied to calculate the shape parameter and the scale parameter through the known parameter, that is the cumulative failure probability. The results are as follows:

$$
\hat{\beta}=1.8536, \hat{\eta}=57662
$$

Thus, the reliability of antiskid valve at each censor time is obtained as Table 4.

Table 4 The results of the cumulative failure probability

\begin{tabular}{cccccc}
\hline$t_{i}(h)$ & 345 & 690 & 1035 & 1380 & 1725 \\
\hline $\mathrm{R}(\mathrm{t})$ & 0.9983 & 0.9950 & 009915 & 0.9872 & 0.9837 \\
$\mathrm{t}_{\mathrm{i}}(\mathrm{h})$ & 2070 & 2415 & 2760 & 3105 & 3450 \\
$\mathrm{R}(\mathrm{t})$ & 0.9808 & 0.9776 & 0.9742 & 0.9725 & 0.9718 \\
\hline
\end{tabular}

According the (19), the curve of the reliability $\mathrm{R}(\mathrm{t})$ can be drawn as Fig. 3. As shown in Fig. 4, it concludes that the reliability of antiskid valve is decreases with time. And the life of the antiskid valve is about $50000 \mathrm{~h}$. And the Mean Time Between Failure (MTBF) is obtained as follows:

$$
M T B F=\int_{0}^{\infty} R(t) d t=51212 h
$$

51212 hours which is equals to about 5.8 years, it fit in with the actual situation.

The failure function of the antiskid valve is:

$$
F(t)=1-\exp \left[-\left(\frac{t}{57662}\right)^{1.8536}\right]
$$

Sensitivity analysis is an optimized way to research the influence that the parameter drawn on the mathematical model. It is an effective way to find the factor that affect the model most. In the optimal method, the sensitivity analysis is used to study the stability of the optimal solution when the original data is uncertain. When processing the hyper-parameter c. It's being subjective to choose the value of $\mathrm{c}$. Thus, the robustness of the hyper-parameter c need to be ensured. That is to make sure the results various little while the value of $\mathrm{c}$ is changed.

According to the index for the project, the cumulative failure probability should be lower than 0.015 at the end of the fifth time censoring test. From the Fig. 4, it can be concluded that the value of hyper-parameter $\mathrm{c}$ has little influence on the cumulative failure probability. Considered of the robustness of the hyper-parameter $\mathrm{c}$, this method is convincible.

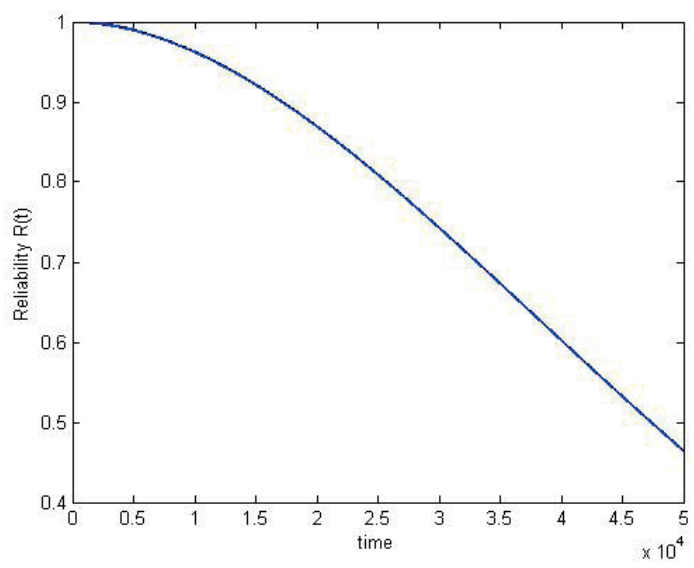

Fig. 4 Probability density function of Beta distribution

According to GO methodology, we choose the type 1 operator simulate the dryer, air cylinder, adjusting pressure valve, emergency braking electromagnetic valve, pressure valve, brake cylinder and mechanical transmission components; type 5 operator to simulate the brake controller, air compressor and other input unit; type 6 operator to simulate the EP valve; type 10 operator to simulate relay valve. The short horizontal line on the left side of the figures represent operator types, the right number represent numbers corresponding to the system unit.

The reliability of each part of the antiskid system can be obtained through the Bayes method. It can be concluded as Table 5. And the reliability parameters of antiskid system can be obtained as Table 6 through GO methodology algorithm.

Table 5 The reliability of parts of antiskid system

\begin{tabular}{lccc}
\hline Serial No. & Element & Failure rate ${ }^{-1}$ & MTTR/h \\
\hline 1 & Brake controller & $4.9736 \mathrm{e}-5$ & 2.0567 \\
2 & Air compressor & $2.7593 \mathrm{e}-5$ & 5.5784 \\
3 & Dryer & $6.3864 \mathrm{e}-6$ & 5.0214 \\
4 & Air cylinder & $1.0854 \mathrm{e}-5$ & 4.9054 \\
5 & Antiskid valve & $1.8060 \mathrm{e}-5$ & 0.1212 \\
6 & Solenoid valve & $2.3228 \mathrm{e}-8$ & 0.5000 \\
7 & EP valve & $1.8090 \mathrm{e}-5$ & 1.5641 \\
8 & Relay valve & $2.5210 \mathrm{e}-5$ & 4.9672 \\
9 & Booster air cylinder & $6.3864 \mathrm{e}-6$ & 5.0214 \\
10 & Brake cylinder & $6.3867 \mathrm{e}-6$ & 1.2000 \\
11 & Disc braking equipment & $1.0412 \mathrm{e}-4$ & 1.7354 \\
\hline
\end{tabular}


Table 6 The reliability parameter of antiskid system

\begin{tabular}{lc}
\hline parameter & value \\
\hline Failure rate $\lambda /\left(10^{4}-1\right)$ & $2.4125 \mathrm{e}-1$ \\
Maintenance rate $\mu /\left(10^{4}-1\right)$ & $4.6706 \mathrm{e}-1$ \\
$\mathrm{MTBF} / \mathrm{h}$ & $4.9256 \mathrm{e}+4$ \\
$\mathrm{MTTR} / \mathrm{h}$ & 2.1214 \\
$\mathrm{MCT} / \mathrm{h}$ & $4.9258 \mathrm{e}+4$ \\
$\mathrm{~A}$ & 0.99956 \\
$\bar{A}$ & 0.00044 \\
Mean failure times at each unit time $\mathrm{f}$ & $2.03 \mathrm{e}-5$ \\
\hline
\end{tabular}

\section{Conclusions}

(1) In this paper, the failure of antiskid system of rail transit vehicle braking system is analyzed. Focus on the analysis of the failure of the antiskid valve which is the core component of the antiskid system. The working principle of the antiskid valve is analyzed, based on this, many possible faults of antiskid valve is listed.

(2) In the process of building the reliability model and choosing the prior distribution for the cumulative failure probability, we make full use of the prior information, adding to the time censoring test data, the estimation of the shape parameter is concluded which is $\hat{\beta}=1.8536>1$, it implies that the failure type of CRH2 EMU follows the wear-out mechanism, and the reliability model which is the Weibull distribution is fit to the actual situation.

(3) This paper provides a certain kind of method to evaluate the reliability of the antiskid valve of CRH2 EMU. It also can be a reference to other kind of valve served in EMU. In particular, the application of MCMC method can lay the foundation of the solution to multidimensional integration with various hyperparameter. Then through the analysis of sensitivity of hyperparameter $\mathrm{c}$, the mathematical model is proved more convincible.

(4) To ensure that obtain the more correct and more invincible result of evaluation through less test data, we need to collect the prior information, and process the information to make it quantification, form the prior distribution, and apply it to the Bayes method.

(5) At last, the reliability of the antiskid system is presented through GO method.

All sections are numbered consecutively except for the Acknowledgement and the References.

\section{Acknowledgement}

This paper was supported by the national natural science fund project (51175028) and the Great Scholars Program (CIT $\&$ tcd20150312).

\section{References}

Li, Y. L. (2015). Open circuit fault analysis and solving measures of EMU speed antiskid valve. Window of High-tech. 11, p. 53. (in Chinese). https://doi.org/10.3969/j.issn.1007-1547.2015.08.047

Liu, P. S. (2001). antiskid control of rail transit vehicle. Railway Vehicle. 7, p. 9. (in Chinese). https://doi.org/10.3969/j.issn.1002-7602.2001.07.003

Niu, Y. C. (2010). Common fault analysis and solution of TFX1 type antiskid valve. Mechanical Management and Development. 6, pp. 79. (in Chinese).

https://doi.org/10.3969/j.issn:1003-773X.2010.06.040

Yao, S. W., Chen, Z. F., Qian, L. X. (2001). Research on intelligent electronic antiskid control system. China Railway Science. 4, pp. 62. (in Chinese). https://doi.org/10.3321/j.issn:1001-4632.2001.06.001

Wang, H. S. (2001). Reliability analysis of locomotive and rolling stock parts based on weighted least square method. Journal of China Railway Society. 6, pp. 21-25. (in Chinese).

https://doi.org/10.3321/j.issn:1001-8360.2001.06.005

Wang, L. Z., Xu, Y. G., Zhang, J. D. (2008). Reliability analysis model and Its Application Research of key components of railway equipment. Journal of China Railway Society. 4, pp. 93-97. (in Chinese). https://doi.org/10.3321/j.issn:1001-8360.2008.04.018

Wu, M. L., Wang, X. Y., Tian, C. (2009). Reliability of Relay Valve of Brake System for Rail Vehicles. Journal of Southwest Jiaotong University. 3, pp. 365-369. (in Chinese). https://doi.org/10.3969/j.issn.0258-2724.2009.03.010 